\title{
An unusual cause of abdominal pain in a male patient: Endometriosis
}

Fadi l. Jabr, Venk Mani

Departments of Internal Medicine and ${ }^{1}$ Pathology, Horizon Medical Center, Dickson, TN, USA

\begin{tabular}{|c|}
\hline Access this article online \\
\hline Website: www.avicennajmed.com \\
\hline DOI: 10.4103/2231-0770.140660 \\
\hline Quick Response Code: \\
\hline
\end{tabular}

\section{ABSTRACT}

A rare case of abdominal pain secondary to endometriosis in a 52-year-old man with cirrhosis is presented. The patient had a repair of recurrent inguinal hernia 7 months prior to presentation. An exploratory laparotomy revealed a $4.5 \mathrm{~cm} \times 2.5 \mathrm{~cm}$ cystic mass attached to the urinary bladder and the inguinal area. The mass pathology showed a cystic lumen of diameter of $1.5 \mathrm{~cm}$ filled with old blood and surrounded by thick muscular mass. Microscopically, the lesion consisted of thick smooth muscle fibers that concentrically surround a central lumen that is bordered by endometrium such as glands and stroma. Immunostains were positive for estrogen receptors and progesterone receptors in the glandular component and for CD10 in the stroma, which is consistent with endometriosis.

Key words: Abdominal pain, endometriosis, male, metaplasia, Mullerian agenesis

\section{INTRODUCTION}

The occurrence of endometriosis in men is extremely rare. Only a few cases of endometriosis in men have been reported in the literature. ${ }^{[1-3]}$ The present case describes a uterine-like mass endometriosis attached to the urinary bladder and extending into the inguinal canal area in a man with a history of cirrhosis.

\section{CASE REPORT}

A 52-year-old man with a history of cirrhosis secondary to hepatitis C. Surgical history revealed banding of esophageal varices and a right inguinal hernia repair twice over the past 2 years. The last repair of inguinal hernia was 7 months ago in which he required mesh placement. He presented to the emergency department with an excruciating stabbing pain in the right lower abdomen and pelvis area for 3 weeks. The pain was worse on getting up from a supine position and was not relieved by bowel movements. It slightly increased upon urination as well. Physical examination revealed moderate tenderness at the lower abdomen, over the urinary bladder, and over the site of the previous inguinal hernia surgery. There were no signs of hernia recurrence. The testicles were descended and nontender. Computed tomography showed hepatic cirrhosis in addition to an extraperitoneal soft tissue of $2.6 \mathrm{~cm} \times 2.3 \mathrm{~cm}$ in the pelvis adjacent to the urinary bladder and extending into the right inguinal canal [Figure 1]. A diagnosis of Meckel diverticulum was initially suspected, but it was later ruled out by a negative radio-nuclear Meckel scan. Blood workup revealed white blood cell count of $8700 / \mathrm{mm}^{3}$, bilirubin of $1.6 \mathrm{mg} / \mathrm{dL}$ and aspartate aminotransferase of $83 \mathrm{U} / \mathrm{L}$. During a laparoscopy, a cystic mass was viewed attached to the urinary bladder and the right inguinal area [Figure 2]. The mass was subsequently excised from the urinary bladder surface and then removed from the site of the previous inguinal hernia repair. The superficial layer of the bladder was then repaired. The mass measured $4.5 \mathrm{~cm} \times 2.5 \mathrm{~cm}$ in diameter. Histological sections demonstrated a cystic lumen of diameter of $1.5 \mathrm{~cm}$ filled with old blood and surrounded by thick muscular mass. When viewed microscopically, the lesion consisted of thick, smooth muscle fibers that concentrically surround a central lumen that was bordered by endometrium-like glands and stroma [Figure 3]. Immunostains were positive for estrogen

Address for correspondence: Dr. Fadi I. Jabr, Department of Internal Medicine, Horizon Medical Center, 111 HWY 70E, Dickson, TN 37055 , USA. E-mail: fijabr@gmail.com 


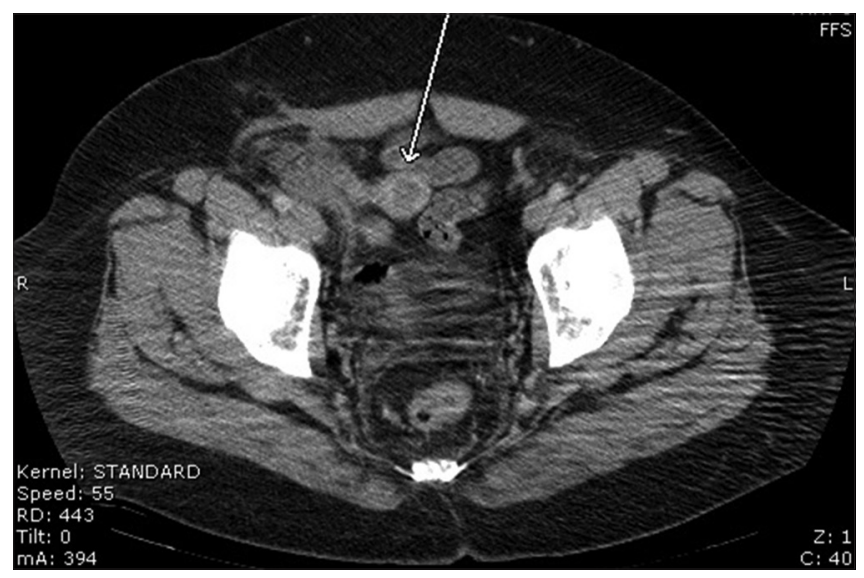

Figure 1: Computed tomography showed an extraperitoneal soft tissue of $2.6 \mathrm{~cm} \times 2.3 \mathrm{~cm}$ in the pelvis adjacent to the urinary bladder and extending into the right inguinal canal

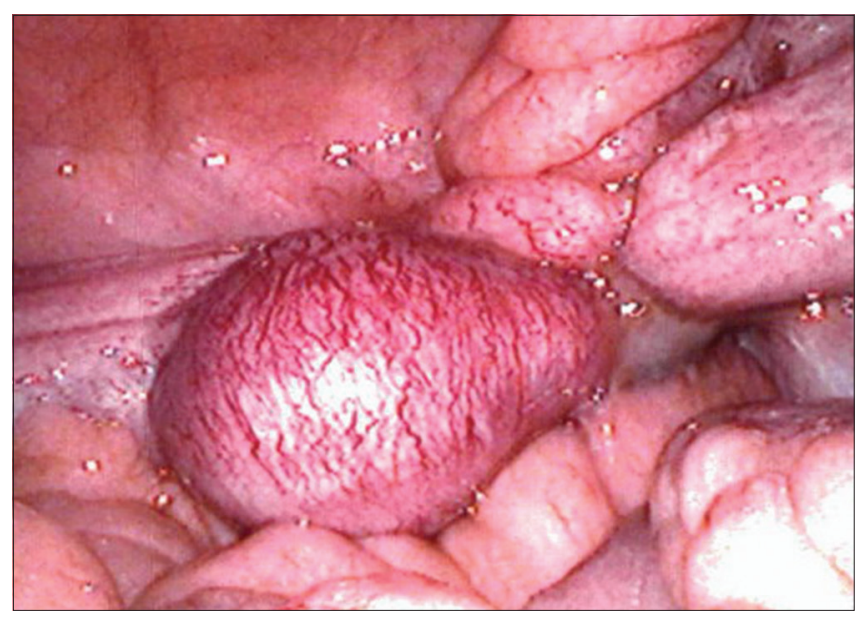

Figure 2: Laparoscopy showed a cystic mass attached to the urinary bladder and the right inguinal area

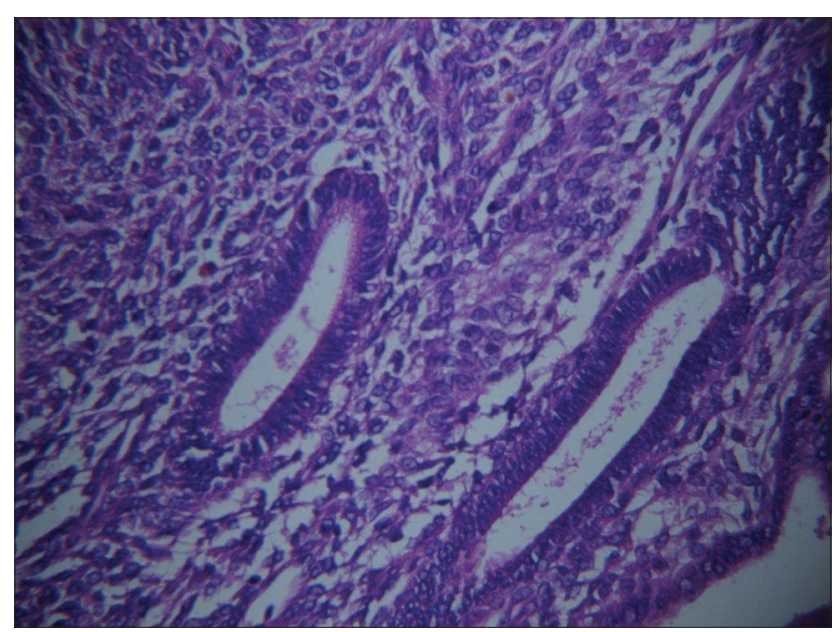

Figure 3: Endometrial type glands and stroma

and progesterone receptors in the glandular component and for CD10 in the stroma, which was consistent with endometriosis. The patient had an uneventful postoperative course and his pain resolved completely.

\section{DISCUSSION}

Endometriosis, by definition, is an ectopic endometrium. Pathogenesis of endometriosis in the female is not well understood, and three theories have been proposed: Transplantation, coelomic metaplasia, and embryologic cell rests. ${ }^{[4,5]}$ The implantation or transplantation theory proposes that the endometrial tissue from the uterus is shed during menstruation and transported through the fallopian tubes to pelvic structures. Transplantation is unlikely the cause of the endometriosis in this patient or any of the male patients obviously because of absence of menstruation. ${ }^{[1,3]}$ The coelomic metaplasia theory states that the cells lining the coelomic cavity or mesothelium of the peritoneum are capable of dedifferentiating into endometrial tissue. ${ }^{[4,5]}$ Under certain inflammatory and hormonal influences, coelomic metaplasia could cause endometriosis in women with Mullerian agenesis and very rarely in men. In induction theory, unknown biological factors, which might be exogenous or released from the endometrium, induce undifferentiated mesenchyma or embryonal rest to form endometriotic tissue. ${ }^{[5]}$ Between the utricle and the appendices testes, cell-rests may persist. ${ }^{[3]}$ These Mullerian cell-rests lie along the course of the ejaculatory and deferent ducts. ${ }^{[1,3]}$ In one of the cases reported in the literature, endometriosis was found along the ductus deferens suggesting that induction theory is a likely mechanism. ${ }^{[3]}$ In that case, the patient was maintained on clomiphene citrate, a selective estrogen receptor modulator that binds estrogen receptors in the hypothalamus and hypophysis. ${ }^{[3]} \mathrm{In}$ two cases, there was a history of prostate carcinoma treated with estrogen for several years. ${ }^{[1,2]}$ One of these patients had a para-testicular multi-lobular cyst. In that patient, pathology revealed stromal endometriosis associated with tunica vaginalis again suggesting Mullerian metaplasia and stromal cell hyperplasia under the influence of prolonged estrogen therapy. ${ }^{[2]}$ The other patient described in that report had endometriosis of the urinary bladder after radical prostatectomy, orchiectomy and prolonged estrogen treatment. ${ }^{[1]}$ Our patient had cirrhosis that is known to be associated with significantly increased estradiol levels. ${ }^{[6]}$ This is explained by peripheral conversion of androgens, namely androstenediones, to both estrones and estradiols. ${ }^{[6]}$ Interestingly, in one case report, the patient was operated on for inguinal hernia 3 times prior to the occurrence of the endometriosis at the same site. ${ }^{[3]}$ Similarly, our patient had two right inguinal hernia repairs within 2 years prior to the development of the endometriosis attached to the right inguinal area. A possible hypothesis is that repeated surgeries for inguinal hernia repair in the setting of high estrogen levels, with or without mesh implants, had 
caused Mullerian metaplasia and stromal cell hyperplasia. Coelomic metaplasia of the mesothelium of the peritoneum to dedifferentiate into endometrial tissue is a less likely mechanism.

In summary, endometriosis attached to the urinary bladder and inguinal area -in a male patient is a very unusual condition. In exceptionally rare occasions, it can present as an acute abdomen in men. Finally, it should typically be treated with surgical resection.

\section{REFERENCES}

1. Pinkert TC, Catlow CE, Straus R. Endometriosis of the urinary bladder in a man with prostatic carcinoma. Cancer 1979;43:1562-7.
2. Fukunaga M. Paratesticular endometriosis in a man with a prolonged hormonal therapy for prostatic carcinoma. Pathol Res Pract 2012;208:59-61.

3. Simsek G, Bulus H, Tas A, Koklu S, Yilmaz SB, Coskun A. An unusual cause of inguinal hernia in a male patient: Endometriosis. Gut Liver 2012;6:284-5.

4. Rock JA, Markham SM. Pathogenesis of endometriosis. Lancet 1992;340:1264-7.

5. Bontis JN, Vavilis DT. Etiopathology of endometriosis. Ann N Y Acad Sci 1997;816:305-9.

6. Gordon GG, Olivo J, Rafil F, Southren AL. Conversion of androgens to estrogens in cirrhosis of the liver. J Clin Endocrinol Metab 1975;40:1018-26.

Cite this article as: Jabr FI, Mani V. An unusual cause of abdominal pain in a male patient: Endometriosis. Avicenna J Med 2014;4:99-101.

Source of Support: Nil, Conflict of Interest: None declared. 